\title{
Putative Corneal Neuralgia Responding to Vitamin D Supplementation
}

\author{
Eric L. Singman David Poon Albert S. Jun \\ Wilmer Eye Institute, Baltimore, Md., USA
}

\section{Key Words}

Vitamin D · Corneal neuralgia $\cdot$ LASIK

\begin{abstract}
A patient with putative corneal neuralgia was incidentally discovered to have hypovitaminosis D. Supplementation of vitamin D appears to have led to a resolution of the patient's pain, whereas other efforts to treat the patient were unsuccessful.

(c) 2013 S. Karger AG, Basel
\end{abstract}

\section{Case Report}

The patient is a 29-year-old female of Indian origin who first reported to our clinic in November 2012 with a month-long complaint of intermittent severe left eye irritation triggered by wearing her soft contact lenses for prolonged periods of time (up to 14-16 $\mathrm{h}$ /day). Symptoms included photophobia and a mild foreign body sensation. She used $V_{\text {Visine }}{ }^{\mathrm{TM}}$ a few times daily and reported that this therapy relieved some of the irritation. She had also noticed that the left eye was often red.

At that visit, the patient's past medical history was significant for gastroesophageal reflux controlled with omeprazole (on an 'as needed' basis). Her past ocular history was significant for the treatment of high myopia with LASIK 6 years prior to the first visit and prophylactic peripheral cryopexy on both eyes. The patient reported enjoying an uneventful postoperative course after LASIK surgery. She was a -10 myope prior to the surgery. In addition, she developed mild myopia 4 years after LASIK surgery, for which she used contact lenses or glasses.

Her family's medical history was significant for high myopia, but her social history was negative for smoking, alcohol or illicit drug use. The patient had no food, drug or environmental allergies. 
With her current correction, the patient's vision was 20/20-2 OD and 20/30-2 OS. Color vision was normal (10 of 10 Ishihara plates correctly scored). The physical exam revealed normal pupillary findings, visual fields by confrontation and extraocular movements. The intraocular pressure (Goldmann) was 8 and 9 in the OD and OS, respectively.

A slit lamp examination revealed mild blepharitis and meibomian gland dysfunction. There were mild conjunctival papillae OU, but neither injection nor lesions of the conjunctiva otherwise. The corneas bilaterally demonstrated mild epithelial punctate staining. The LASIK flap scar was bilaterally visible and the flaps were well positioned. The anterior chambers, irides and lenses were bilaterally normal.

Dilated fundoscopic exam bilaterally confirmed normal optic nerves, each with a cup to disc ratio of 0.5 , as well as normal vitreous and maculae. There were areas of far peripheral retinal findings consistent with atrophy and previous cryopexy.

The patient was diagnosed with contact lens overwear in the left eye and was instructed to use preservative-free artificial tears up to 6 times/day and artificial tear ointment at bedtime as well as to decrease contact lens use to a maximum of $6-8 \mathrm{~h} /$ day. The patient was offered a follow-up appointment in 1 month if still symptomatic or after 6 months if not. Corneal topographic measurements were suggested for the follow-up visit.

The patient returned to our clinic 3 months later with continuing complaints of severe left eye irritation, worse in the wind. She completely discontinued contact lens wear 2 weeks prior to this visit, but suffered persistent left eye pain and blurred vision. She reported that the left eye had been red and that she had been told that she sleeps with her eyes 'slightly open'.

Her medical history changed. She reported that she was undergoing an endocrinologic workup because she noted hair loss, one episode of galactorrhea and 4 days of spotting outside the time of her usual menses. We also learned that her mother was treated for hypothyroidism.

The patient's family doctor sent a battery of blood tests, including a pregnancy test, transferrin/Fe/TIBC, vitamin B-12, RBC folate, thyroid-stimulating hormone, DHEA-S, free testosterone, prolactin, ferritin, ANA, anti-hepatitis B surface antigen antibody, hepatitis C antibody screen, hepatitis B surface antigen, Treponema pallidum antibody, vitamin D (25$\mathrm{OH}), \mathrm{D} 2, \mathrm{D} 3$, luteinizing hormone, follicle-stimulating hormone, complete blood count with differential and HIV antibody test. The anti-hepatitis B antibody was positive, consistent with the patient's history of vaccination. Free testosterone was slightly elevated. The folliclestimulating hormone and luteinizing hormone were considered within normal range for a woman during the luteal phase of her menstrual cycle. Vitamin D 25-OH was $15 \mathrm{ng} / \mathrm{ml}$; the normal range is between $30-100 \mathrm{ng} / \mathrm{ml}$, indicating a deficiency in our patient. The patient was told that vitamin D deficiency is common among people of the Indian ethnicity.

Her vision at this second visit with correction was 20/25 OD and 20/40 OS, improving with pinhole to $20 / 20$. The remainder of the physical exam was unchanged, including the presence of mild blepharitis, meibomian gland dysfunction and punctate staining on both corneas.

The patient was brought to the attention of our corneal service, where she was examined and diagnosed with possible exposure keratopathy. Notably, the findings between the two eyes were identical, while the patient confirmed that the complaints were only in the left eye. The patient was instructed to continue aggressive lubrication with preservative-free artificial tears 6-8 times/day and was prescribed erythromycin ointment 4 times/day.

We remained in communication with the patient. She reported that she tried oral nonsteroidal anti-inflammatory medication for the pain, but this did not help. She added that the lubricating eye ointment at bedtime helps with the morning dryness, and sleeping later than 
usual exacerbates this feeling. In addition, she felt that the additional lubrication helped the blurred vision OS. However, the patient reported that the burning pain in the left eye persisted and questioned whether her vitamin D deficiency could be the problem. Thereafter, the patient started taking oral vitamin D supplementation, 1,000 IU/day and within 4 days reported being completely free of the burning pain. We remained in e-mail communication with the patient; at 4 weeks, she was much more comfortable and no longer needed eye drops. Three months after her last visit, she was still free of pain without topical therapy and could even tolerate wearing her contact lenses for short periods of time, but only if she used topical lubrication. Although the patient returned to her hometown 3 months after her last visit, a recent e-mail 5 months after her last visit confirmed that her situation remained improved. The patient returned to her hometown in May and we were not able to schedule a final follow-up. Her family doctor did not have the opportunity to obtain a follow-up serum vitamin D level.

At this point, we made a putative diagnosis of corneal neuropathic pain. This condition has been described by patients as burning, stinging, scratchiness or just pain, and this pain is usually severe [1]. It can mimic symptoms of dry eye and blepharitis, and the objective findings at the slit lamp are disproportionately benign compared to the complaints; patients seem to not respond to topical therapies as it appeared to be the case in our patient. Hyperesthesia with abnormal sensitivity to wind or light can be reported by patients, including our patient. Therapies that have been recommended for this condition include lubrication, anticonvulsants, tricyclic antidepressants and serotonin reuptake inhibitors, a scleral reservoir lens and evaporation-prevention goggles.

Corneal neuralgia has also been reported in patients after refractive surgery [2], although we do not presume to suggest that our patient's pain was related to her relatively remote history of LASIK.

A limitation of this report is that we did not have the opportunity to examine our patient again once her symptoms resolved. In addition, we do not know the degree to which the vitamin D supplementation reversed the deficiency discovered by serum testing. We did note that our patient's exam findings were extremely mild and that they did not change between her two visits despite her good compliance with suggested interventions. We believe it is also noteworthy that the patient was able to discontinue all topical lubrications soon after the vitamin D therapy was initiated.

Notably, there is a published case report indicating that correction of vitamin D deficiency reversed diabetic neuropathic pain [3]. Furthermore, low levels of vitamin D have been shown to be an independent risk factor for diabetic neuropathy $[4,5]$. In patients suffering from Sjögren's syndrome, neuropathy has been associated with low levels of vitamin D [6], and in pediatric patients with unexplained limb pain, vitamin D insufficiency has been reported frequently [7].

It is unclear how vitamin D might be associated with the relief of pain. It has been reported that hypovitaminosis D causes hyperinnervation of nociceptors in skeletal muscle tissue [8]. Vitamin D also reduces nitric oxide production [8]; nitric oxide is believed to be a nociceptive transmitter and cause nociceptive hypersensitivity [9].

The metabolism of vitamin D in the cornea is not completely understood. Calcitriol (the active form of vitamin D), calbindin D-28k (a vitamin D-dependent calcium-binding protein) and a plasma membrane calcium pump have been immunolocalized to the human corneal endothelium and basal epithelium [10]. In addition, calbindin 2 is reported to be expressed in the corneal epithelium, stroma and endothelium [11]. Vitamin D metabolites were found in human corneal limbal epithelial cells after irradiation with UV-B, suggesting that these 
Singman et al.: Putative Corneal Neuralgia Responding to Vitamin D Supplementation

cells were capable of synthesizing these products [12]. Finally, vitamin D has been shown to enhance corneal epithelial barrier function [13].

We recognize that our diagnosis of corneal neuralgia for our patient was a putative one. In addition, pain is subjective and we cannot rule out a placebo effect. However, given the literature regarding neuropathic symptoms associated with vitamin D deficiency, it may be reasonable to consider assessing vitamin $\mathrm{D}$ levels and appropriately supplementing deficiencies thereof in patients who present with recalcitrant corneal/ocular surface pain with disproportionately mild findings on clinical examination.

\section{References}

1 Rosenthal P, Borsook D: The corneal pain system. Part I: the missing piece of the dry eye puzzle. Ocul Surf 2012;10:2-14.

2 Nettune GR, Pflugfelder SC: Post-LASIK tear dysfunction and dysesthesia. Ocul Surf 2010;8:135-145.

-3 Bell DS: Reversal of the symptoms of diabetic neuropathy through correction of vitamin D deficiency in a type 1 diabetic patient. Case Rep Endocrinol 2012;2012:165056.

-4 Shehab D, Al-Jarallah K, Mojiminiyi OA, Al Mohamedy H, Abdella NA: Does vitamin D deficiency play a role in peripheral neuropathy in Type 2 diabetes? Diabet Med 2012;29:43-49.

-5 Soderstrom LH, Johnson SP, Diaz VA, Mainous AG 3rd: Association between vitamin D and diabetic neuropathy in a nationally representative sample: results from 2001-2004 NHANES. Diabet Med 2012;29:50-55.

-6 Agmon-Levin N, Kivity S, Tzioufas AG, López Hoyos M, Rozman B, Efes I, Shapira Y, Shamis A, Amital H, Youinou P, Shoenfeld YJ: Low levels of vitamin-D are associated with neuropathy and lymphoma among patients with Sjögren's syndrome. J Autoimmun 2012;39:234-239.

7 Rokyta R, Fricová J: Ontogeny of the pain. Physiol Res 2012;61:S109-S122.

8 Bartley J: Post herpetic neuralgia, schwann cell activation and vitamin D. Med Hypotheses 2009;73:927929.

-9 Tegeder I, Scheving R, Wittig I, Geisslinger G: SNO-ing at the nociceptive synapse? Pharmacol Rev 2011;63:366-389.

-10 Johnson JA, Grande JP, Roche PC, Campbell RJ, Kumar R: Immuno-localization of the calcitriol receptor, calbindin-D28k and the plasma membrane calcium pump in the human eye. Curr Eye Res 1995;14:101-108.

11 Jirsova K, Neuwirth A, Kalasova S, Vesela V, Merjava S: Mesothelial proteins are expressed in the human cornea. Exp Eye Res 2010;91:623-629.

-12 Lin Y, Ubels JL, Schotanus MP, Yin Z, Pintea V, Hammock BD, Watsky MA: Enhancement of vitamin D metabolites in the eye following vitamin D3 supplementation and UV-B irradiation. Curr Eye Res 2012;37:871-878.

-13 Yin Z, Pintea V, Lin Y, Hammock BD, Watsky MA: Vitamin D enhances corneal epithelial barrier function. Invest Ophthalmol Vis Sci 2011;52:7359-7364. 